\title{
A public health timeline to prepare for COVID-19 vaccines in Canada
}

\author{
Noni E. MacDonald ${ }^{1}$ (D) $\cdot$ Jeannette Comeau ${ }^{1} \cdot$ Eve Dubé $^{2} \cdot$ Lucie Bucci $^{3} \cdot$ Janice E. Graham $^{1}$
}

Received: 13 July 2020 / Accepted: 17 September 2020 / Published online: 5 November 2020

(C) The Canadian Public Health Association 2020

\begin{abstract}
For control of COVID-19, community immunity is required, necessitating widespread immunization. COVID-19 vaccines are coming to Canada, with the government announcing in August 2020 agreements with four different companies for their COVID19 vaccine if their trials are successful. Never before has public health had to rapidly develop a vaccine introduction program for multiple new but differing vaccines with the added pressure that the program is needed across all ages and in all Canadian communities and there is high probability of not enough vaccine to go around at the start. Traditional public health vaccine introduction planning will need to be both accelerated and more comprehensive to ensure optimal uptake across the country. This overview highlights a number of points for consideration by public health in their planning for COVID-19 vaccines before these COVID-19 vaccines are available, once they are available, once supplies are plentiful, and throughout the vaccine program. Targeted and tailored communications are key elements needed to reach and positively influence diverse communities, regions, ages, languages, education levels and lived experiences.
\end{abstract}

\section{Résumé}

Pour contrôler la pandémie de la COVID-19, une immunité de groupe est requise, ce qui nécessite de la vaccination à grande échelle. Des vaccins contre la COVID-19 seront bientôt disponibles au Canada, le gouvernement ayant annoncé en août 2020 des ententes pour obtenir les vaccins de quatre compagnies différentes si leurs essais sont couronnés de succès. Jamais auparavant la santé publique a eu à préparer rapidement l'introduction d'un nouveau programme avec plusieurs nouveaux vaccins, sans compter la pression supplémentaire liée au fait que le programme visera tous les groupes d'âges, devra rejoindre l'ensemble des communautés canadiennes et qu'il n'y aura probablement pas suffisamment de vaccins pour tous au moment de démarrer le programme. Les stratégies de santé publique pour l'introduction de programme de vaccination devront à la fois être accélérées et plus détaillées pour assurer une couverture vaccinale optimale à travers le pays. Cet article survole différents éléments à considérer par les autorités de santé publique dans la planification des programmes de vaccination contre la COVID-19 avant que les vaccins soient disponibles, lorsqu'ils le seront, lorsque l'approvisionnement en vaccins sera abondant et à travers le programme de vaccination. Des communications adaptées et ciblées sont des éléments essentiels pour rejoindre et influencer positivement les Canadiens provenant de différentes communautés et régions et ayant différents âges, langues maternelles, niveaux de scolarité et expériences.

Keywords COVID-19 vaccines $\cdot$ COVID-19 immunization $\cdot$ Public health $\cdot$ Pandemic control $\cdot$ Communication

Mots-clés Vaccins contre la COVID-19 • Vaccination contre la COVID-19 · Santé publique · Contrôle de la pandémie · Communication

Noni E. MacDonald

noni.macdonald@dal.ca

1 Department of Pediatrics, Dalhousie University, IWK Health Centre, Halifax, Nova Scotia, Canada

2 Institut National de Santé Publique du Québec and Université Laval, Québec, Québec, Canada

3 Immunize Canada, Canadian Public Health Association, Ottawa, Ontario, Canada

\section{Introduction}

As COVID-19 shines a light on the need for global governance mechanisms to strengthen economies and health, the compelling role of vaccines has never been clearer. Learning from Ebola (Pltokin et al. 2015), a host of publicly funded research consortia, institutes and pharmaceutical manufacturers such as the Coalition for Epidemic Preparedness Innovations (CEPI) (https://cepi.net/ covid-19/) are working aggressively to develop effective vaccines 
(Lurie et al. 2020). Vaccine manufacturing plans while vaccines are still in development (Hosangadi et al. 2020), along with a new multibillion dollar fund to subsidize COVID-19 vaccine for lower income countries (Usher 2020), are unfolding. The World Health Organization Strategic Advisory Group of Experts on immunization has provided a values framework for the allocation and prioritization of COVID-19 vaccination globally (World Health Organization, Strategic Advisory Group of Experts on Immunization 2020). Potential trip wires on the road to public availability and uptake of a COVID-19 vaccine in Canada need to be thought through in preparation for vaccine rollout.

The COVID-19 vaccine introduction and rollout will be a unique experience for public health. Never before has public health had to rapidly develop a vaccine introduction program for multiple new but differing vaccines with the added pressure that the program is needed across all ages and in all Canadian communities and there is high probability of not enough vaccine to go around at the start.

In the past, introduction of a new vaccine for a newly designated disease has involved a single vaccine type (e.g., HPV vaccine in 2006) or two "similar" vaccines (e.g., rotavirus in 2006) (https://www.cpha.ca/immunization-timeline) for a narrowly defined age group. In contrast, there are over 120 COVID-19 vaccines in development utilizing seven very different platforms and delivery systems (killed, live attenuated, non-replicating adenovirus vector, protein subunit, replicating virus vector, mRNA and DNA) (Lurie et al. 2020). In late August, Canada announced agreements with four different companies for their COVID-19 vaccine if their trials are successful (https://pm.gc.ca/en/news/news-releases/2020/08/31/ new-measures-ensure-supply-future-vaccines-and-therapiesagainst). Thus, more than one type of COVID-19 vaccine will likely become available in Canada. These four vaccines differ from each other in vaccine type/delivery system and are likely to differ in dosing, effectiveness, safety, duration of protection profiles as well as storage requirements. Anxiety and confusion has already arisen in Canada because a phase 3 COVID-19 vaccine clinical trial (not in the four Canada has agreements with) was paused due to a serious adverse event.

Traditional public health vaccine introduction planning will need to be both accelerated and more comprehensive for optimal uptake across the country as COVID-19 vaccines are approved and become available.

This overview highlights a number of points for consideration by public health in their planning for COVID-19 vaccines (see Table 1 for summary).

\section{Before the COVID-19 vaccines are available: start planning now}

Uncertainty is a key feature of the COVID-19 pandemic, making planning the rollout of vaccines complex.

\section{Listen to the community}

In contrast to previous new vaccines targeting limited groups, COVID-19 vaccines will be needed everywhere and for all ages. Public health needs to be on the ground, listening to voices across diverse communities, ages, locales, socio-economic backgrounds, religious affiliations, etc., for concerns being raised about the safety and effectiveness, and the role of vaccines in the control of COVID19 (Ryan et al. 2019). Views and needs of vulnerable and hard-to-reach groups must not be overlooked. COVID-19 concerns are raised in the traditional media and on social media. Disinformation on COVID-19 is rampant (Cornwall 2020). Surveys of the public and social media

Table 1 A public health timeline to prepare for COVID-19 vaccines in Canada

Before COVID-19 vaccine(s) are available, public health needs to:

1) Determine communities' COVID-19 vaccine perspectives and concerns.

2) Develop a communication plan for the range of COVID-19 vaccines potentially available in Canada; address misinformation and tailor the plan to fit different communities. A vaccine crisis communication plan is also needed.

3) Educate all health care professionals on optimization of vaccine acceptance strategies and update their COVID-19 vaccine information.

4) Ensure quality surveillance programs for COVID-19 infections, including detection of vaccine failures and serious Adverse Events Following Immunization (AEFIs), are in place.

Once COVID-19 vaccines are available, public health needs to inform the public:

5) Available COVID-19 vaccines rigorously tested and data reviewed; who to receive, why and where.

6) Infection-control needs addressed.

7) Centralized documentation.

Once COVID-19 vaccine supplies are plentiful:

8) Expanded delivery sites.

Throughout COVID-19 vaccine program, public health needs to:

9) Document processes and lessons learned and share observations.

10) Continue routine immunization. 
listening are key to better inform tailored communication to ready the public for COVID-19 vaccines. Good communication starts with listening, engaging with communities and understanding their information needs and concerns (Bahri and Melero 2018). Trust needs to be earned through opening data and dialogue across communities, using appropriate communication techniques that optimize understanding and tools that are fit for purpose, i.e., chosen because they are likely to reach intended target audience. Listening must be ongoing and not perceived as "one-off political optics" in order to respond with evidence in different contexts when sentiments and flash points shift. Ongoing and responsive listening helps assess how well public health messages are being understood and accepted.

\section{Prepare a communication plan for COVID-19 vaccines, expect changes and manage uncertainty}

The World Health Organization has guidance on risk communication during emergencies that has much that is applicable to COVID-19 vaccine planning, especially around managing uncertainty (https://www.who.int/publications/i/item/ communicating-risk-in-public-health-emergencies).

\section{COVID-19 vaccines and target populations}

COVID-19 vaccine communications will need to reach and positively influence diverse communities, regions, ages, languages, education levels and lived experiences. The targeting and modes of communication to prioritize those communities most affected by COVID will matter since vaccine availability will not likely meet initial demand. In preparing for this supply side issue, public health needs to be open and transparent about how priority groups will be determined, who makes these decisions, and the factors and evidence upon which they are based. Expectation of changing knowledge about available COVID-19 vaccines must be established, with differences in dose schedule, length of protection, efficacy and age group and side effects anticipated. While protecting against serious disease when exposed to COVID-19, some of these vaccines may not prevent mild infection and/or viral shedding. Vaccine recipients, if infected, could spread the virus to others in spite of being immunized. This must be communicated well to manage expectations.

Coherent communication across the country will be key in preparing the public to navigate this complex landscape. Each province and territory presenting different information on the same vaccine, as occurred in Canada with HPV vaccine (Steenbeek et al. 2012), will lead to confusion and undermine trust. The federal and provincial/territorial public health agencies need co-ordination and unity in messaging about each specific vaccine as it becomes available in Canada. National leadership, collaboration with the provinces and territories, as well as within jurisdictions from health unit to health unit, need to be set in place now. Core content on the available vaccines must be similar, although what and how messages are conveyed may vary by community targeted, i.e., message tailoring (Dubé et al. 2020). Uncertainties about vaccines in general and their specifics need to be acknowledged - the science of what is known, what is not known, and what is being done to close those gaps in order to nurture individual and community trust in the program and in these vaccines needs to be conveyed.

\section{Complexity, uncertainties and disinformation}

The public will need to be made aware of the complexity of the COVID-19 vaccine program and where to find trustworthy quality information. For example, as vaccines become available, high-quality specific vaccine-relevant information flyers and online information will be needed for health care professionals (HCPs) and for the public with timely updates as more evidence accrues. Flyers, given out by HCPs, coupled with motivational interviewing (see below), have been shown to be helpful in increasing vaccine acceptance (Dempsey et al. 2018).

The impact of misinformation and disinformation must be minimized through a public awareness campaign that supports the incremental development of critical thinking skills (Cornwall 2020; MacDonald and Dubé 2020). Drawing attention to factors influencing disinformation, such as profit, political sparring and advantage, social or psychological reasons, or perpetuation of a hoax (power) (Celliers and Hattingh 2020), can help inoculate against misinformation (van der Linden et al. 2017). Anti-COVID vaccine voices are already surfacing. Emphasizing the techniques used in disinformation can help protect against it (MacDonald and Dubé 2020; Schmid and Betsch 2019). In crafting messages, stories often have a greater impact than statistics (Shelby and Ernst 2013) and more than numbers alone. Anticipated regrets about consequences of a wrong decision also influence acceptance (Sadique et al. 2013) and framing of messages matters (Mostafapour et al. 2019). Optimizing COVID-19 vaccine acceptance to achieve community immunity is critical to protect those with underlying immune deficiency and poor immune response. Uptake levels possibly in the $70-80 \%$ range will be required (Randolph and Barreiro 2020). For each person to be safe, families, communities, countries and the world need to be safe from this virus - with this best achieved through immunization.

\section{Vaccine crisis communications}

All immunization programs need crisis communication plans in order to rapidly and effectively address an event that may undermine trust in a specific vaccine or the immunization program. The European Region of the World Health Organization has a template for preparing in advance for such 
vaccine safety crises (https://www.euro.who.int/_data/ assets/pdf_file/0007/187171/Vaccine-Safety-Eventsmanaging-the-communications-response.pdf). Surveillance for serious adverse events following immunization (AEFI) with rapid investigation and causality assessment (MacDonald and Law 2017) is essential (see section on AEFI below). Given the novelty of the COVID vaccines and their platforms, the range of AEFI will not be known until the vaccines are used extensively. A transparent rapid AEFI response plan with crisis communication embedded is critical in advance or the COVID-19 vaccine program may be easily derailed, also undermining trust in the routine immunization program (MacDonald et al. 2020b). If these events occur with a first dose, and a second dose is scheduled, advice might be sought from local immunization experts such as the network of "Special Immunization Clinics" across Canada (Top et al. 2016), albeit these are for pediatric patients only. An adult equivalent is needed for COVID-19 and other vaccines in development for older populations.

\section{Train and prepare health care professionals}

\section{COVID-19 vaccine information and techniques to optimize acceptance}

Given the key role HCPs play in vaccine decision-making and acceptance (Dawson et al. 2018), COVID-19 vaccine education for all HCPs is essential, as is keeping them current as new COVID-19 and COVID-19 vaccine information accrues. HCPs are not only a vaccine target group themselves, they are critical in educating the public. HCPs need training to answer questions that will arise both in their health care work settings as well as with their families and friends. They will need to have information at the ready, know how to optimize acceptance of COVID19 vaccines once available, and where people can be immunized. Motivational interviewing techniques for immunization need to be taught to improve vaccine acceptance and build trust (Dempsey et al. 2018; Gagneur et al. 2018) (see also https://canvax.ca/brief/motivationalinterviewing-powerful-tool-immunization-dialogue). Training will also be needed on how to prevent immunization stress-related responses (ISRR) in order to make the immunization experience more positive (Gold et al. 2020) (see AEFI section below). For HCPs to foster public trust in the COVID-19 vaccines, they will need to ensure people understand and accept uncertainty that "we are building our boat and sailing it at the same time".

Explaining the complexity surrounding COVID-19 vaccines should be possible within a few months. The general public in Canada has learned to speak knowledgably about flattening the epidemic curve and many now understand the concept of $\mathrm{R} 0$, the basic reproductive number, i.e., the expected number of cases directly arising from one case in a population where all individuals are susceptible to infection. HCPs through their interactions with patients and others need to build on what those in their communities know. Building trust is critical. Trust requires not only compassion and caring but also that HCPs are up to date in their COVID-19 disease and vaccine knowledge, i.e., are seen as competent. Having all HCPs providing similar messages can help decrease patient and general public confusion. Public health can further support HCPs in this role by having many voices (scientists, academics, community leaders, etc.) speaking up in support of the safety and effectiveness of these new vaccines once they are available.

\section{COVID-19 immunization consent}

As with any health care visit, informed consent for COVID-19 immunization must be conducted in conversational dialogue, without coercion, and be experienced as genuine (https:// ethics.gc.ca/eng/policy-politique_tcps2-eptc2_2018.html). Given that the COVID-19 vaccines are new and differ among themselves, and what is available in Canada may change over time, the consent process has some added complexity. HCPs will need to know that in addressing concerns while engaged in the consent process, overemphasis on potential and unknown risks may undermine acceptance. Thus, HCPs will need training on what needs to be included and on how to openly address issues of concern, including those about safety and uncertainty (see motivational interviewing above). HCPs and patients will need to know the specific COVID-19 vaccine being given, its schedule requirements if more than one dose is needed, how long protection is expected to last, what are known common adverse events (e.g., sore arm, mild fever), and how to address these and report on unexpected events (i.e., passive AEFI surveillance). Confusion with information on other COVID-19 vaccines must be minimized. Patients will need to be queried about underlying diseases or other factors that might alter response. Pre-visit opportunities (online, virtual or phone visits) might be considered to educate about the specific vaccine, discuss consent, and book patient visits to speed this process along as urgency demands.

\section{Ensure surveillance programs for detection of COVID- 19 infection and serious adverse events following immunization}

An AEFI is classified as serious if it results in death, is life threatening, requires hospitalization or prolongation of existing hospitalization, results in persistent or significant disability/incapacity, is a congenital anomaly/birth defect or requires intervention to prevent permanent impairment or 
damage (https:/www.who.int/vaccine_safety/publications/ gvs_aefi/en/ page 9). Serious AEFI can undermine trust in a program, especially a new vaccine program. Not every serious AEFI is due to the vaccine. The event may be coincidental. Expert causality assessment of case information on the AEFI is required to make this decision.

Given that vaccine safety concerns are commonly advanced for not accepting vaccination (Lane et al. 2018), it is important that serious AEFI following COVID-19 vaccines are detected and assessed to determine causality. Thus, strong national surveillance programs to detect vaccine failures (COVID-19 disease in vaccine recipients) and rare side effects not noted in the pre-approval studies need to be in place. In Canada, there is an excellent system for detecting COVID-19 disease and one active surveillance system for detecting serious AEFI (i.e., required hospitalization) (Bettinger et al. 2014). To detect vaccine failures, it will be critical that for all patients tested for COVID-19, details on receipt of any COVID-19 vaccine are collected alongside usual information, i.e., what specific vaccine, when, where. With respect to active AEFI surveillance, IMPACT: the Canadian Immunization Monitoring Program, ACTive, only searches among children's hospitals, not adult hospitals (Bettinger et al. 2014). A similar system of active surveillance for serious events following immunization among adult vaccine recipients, including seniors, pregnant women and those with underlying medical conditions, is required. The CIRN Serious Outcomes Surveillance (SOS) Network, developed to determine influenza vaccine effectiveness in Canada (Nichols et al. 2018), has been adapted to detect and follow serious COVID-19 infections and might be adapted further, with additional funding, to also search for serious AEFIs post COVID-19 vaccines. Passive reporting of AEFIs is not enough given the millions of patients who will receive these vaccines. A patient-centred fully integrated health information system would make this fairly easy to do (see documentation below).

\section{When COVID-19 vaccines are available, public health needs to inform the public}

\section{Available COVID-19 vaccines rigorously tested and reviewed; who to receive, why and where}

When the first COVID-19 vaccine is available, the public will need to know which one, for whom is it recommended and, if this has changed from earlier messages, why. It will be critical to remind the public that more than one vaccine type is expected in Canada over time and what will be happening in their region. Transparency is critical for trust. The public will also need to be reminded that each of the COVID-19 vaccines available in Canada will have gone through all the rigorous steps to ensure vaccine safety and efficacy, i.e., that all of
Canada's vaccine safety steps have been followed (MacDonald and Law 2017), no corners have been cut, and the trials data have been thoroughly reviewed.

Where vaccine is to be delivered and by whom needs advance planning. If relatively large amounts are available in the first tranche, the venues, supply control and communication strategies may be quite different than if the supplies are meagre. Sites for immunization, how these will be managed and how queue jumping will be prevented need to be communicated transparently. Public Health and Ministries of Health need to be ready and able to respond to private interests trying to buy vaccines and/or queue jump as happened with the 2009/10 H1N1 vaccine for that pandemic. Careful monitoring of how messages are being received and what concerns are being raised will be critical for ongoing message adjustment as the vaccine program rolls out.

\section{Infection-control needs addressed}

Experience so far has shown that COVID-19 infections ebb and flow depending on local control practices. The community context when COVID-19 vaccine becomes available will thus vary across the country and even within a province. Context influences perception of need to be immunized as well as comfort in coming forward to be immunized, i.e., perception of risk of getting COVID-19 in the community and perception of risk while at the immunization venue. Regardless of the venues for vaccine delivery, attention is needed to ensure appropriate infection control practices for the safety of the health care worker and the public. Some potential recipients may be reluctant to come to the venue if they see it as risky in terms of exposure to others who might be infected with COVID-19 (MacDonald et al. 2020a; https:// www.canvax.ca/brief/covid-19-potential-impact-vaccinepreventable-diseases-canada).

\section{Centralized documentation}

Specifics of the vaccine used and visit information must be fully recorded. Documentation is crucial to track who has been immunized with what COVID-19 vaccine to what outcome, i.e., vaccine failures as noted above and serious AEFI. Stand-alone immunization registries may complicate effective outcome monitoring as they lack all needed patient health care information for tracking outcomes as not linked to other health care system interaction data. Patient-centred fully integrated health information systems (Graven et al. 2013) are much more helpful. Immunization registries may be helpful for checking whether other routine immunizations are missing. They also are often unhelpful in noting who is not immunized, i.e., who is missing. Nor are they useful in determining who is at high risk for serious COVID-19 infection, i.e., those who need to move to the 
front of the queue when vaccine supply is limited. Even the size of these populations is not easily known across Canada due to Canada's fractured discontinuous health information systems. While tracking a reported serious COVID-19 AEFI back to the specific vaccine, including lot number, site of delivery, HCP who administered the vaccine, and noted events at the time, as well as being able to quickly access information on others immunized in the same session or with the same lot is possible with some immunization registries, Canada's piecemeal discontinuous information systems make it almost impossible to see how many others immunized and not immunized had similar clinical diagnoses. This makes causality assessment to determine whether the reported serious AEFI is vaccine-related or coincidental more difficult.

Fully integrated patient-centred health information systems are not expensive and do not require complex training to use. Still, there is often pushback from those with vested interests in the current discontinuous systems. Given that millions of Canadians will be immunized, the relative low cost of a fully integrated patient-centred system could be easily justified through the significant improvement in mortality and cost savings when disease management protocols are embedded-which is not possible in our discontinuous systems. The time is right to nationally fix this gap in our provincial and territorial health care systems. This patient-centred fully integrated system can also be used to track COVID-19 diseases, clinical outcomes, interventions, risk factors, etc., with no need for yet another stand-alone discontinuous component for COVID-19 health information.

\section{When COVID-19 vaccines are abundant in Canada}

\section{Expanded delivery sites}

Once supplies of COVID-19 vaccines are stabilized, mass campaigns may be contemplated beyond the traditional delivery venues of clinics, physician offices and pharmacies. These might include schools, universities, colleges, large worksites and long-term care facilities, as well as non-linked sites such as malls, shopping centres, convention centres or arenas. In considering mass campaigns, a number of factors must be borne in mind-informed consent and the ability to document and ensure a quality immunization experience must continue since program errors and immunization stress-related responses (ISRR) are more likely to occur in non-traditional immunization settings (Gold et al. 2020; Hampton 2020). Public health needs to ensure that all HCP immunizing in these sites are trained on how to prevent program errors (Hampton 2020; Hibbs et al. 2015) and ISRR (Gold et al. 2020). Infection control measures are also critical, including avoidance of long waits. Drive-through options might be possible for some if consent information has been discussed in advance virtually (see above). The major advantage of mass campaigns is the number of people immunized in a relatively short time period - an important asset in efforts to control a pandemic.

\section{Throughout COVID-19 program development and implementation}

\section{Document processes and lessons learned}

Throughout COVID-19 vaccine development and implementation, from now until adequate immunization to have community immunity has been achieved, cataloguing what processes worked and what did not work for which contexts and subgroups is important. More pandemics will come. Think through the lessons learned. These findings need to be shared within public health and across health sectors but also with the public, as all have been affected by COVID-19 in one way or another. The manner in which immunization controls the virus must be shared to strengthen community support for subsequent development.

\section{Continue routine immunization}

Beyond attention to the COVID-19 vaccine program, public health must not lose sight of the importance of ensuring high uptake of routine immunizations (MacDonald et al. 2020a). Not ensuring high routine immunization uptake across the age span because of attention diverted to COVID-19 will only compound the calamity and negative health outcomes during the pandemic and later. Routine immunization saves lives and prevents many hospitalizations and much morbidity. Catching up those whose routine immunization has been delayed because of COVID-19 impacts on the health system must be a high priority (MacDonald et al. 2020a).

\section{Conclusions}

COVID-19 has provided us with an opportunity to make Public Health shine with proven results that will better counter future pandemics. Now is the time to prepare for the public health program for COVID-19 vaccines, a more complex task than for any new vaccine ever undertaken in the past. Some components would best be coordinated across the country so that confusion arising from differences in messaging and practices can be avoided. Other aspects, such as best delivery options for different communities, will need to be tailored to fit local culture, context and resources. Given that $70-80 \%$ vaccine acceptance may be needed to achieve community 
immunity, extra attention must be given to ensuring equity of access and special attention to the vulnerable. Time, resources, creativity, intersectoral collaboration and cooperation are all needed. We cannot fail in our task-widespread uptake of COVID-19 vaccination is our lifeline for COVID-19 pandemic control.

\section{Compliance with ethical standards}

Conflict of interest The authors declare that they have no conflicts of interest.

\section{References}

Bahri, P., \& Melero, M. C. (2018). Listen to the public and fulfil their information interests - translating vaccine communication research findings into guidance for regulators. British Journal of Clinical Pharmacology, 84(8), 1696-1705.

Bettinger, J. A., Halperin, S. A., Vaudry, W., Law, B. J., \& Scheifele, D. W. (2014). The Canadian immunization monitoring program, ACTive (IMPACT): Active surveillance for vaccine adverse events and vaccine-preventable diseases. Canada Communicable Disease Report, 40(Suppl 3), 41-44.

Celliers, M., \& Hattingh, M. (2020). A systematic review on fake news themes reported in literature. Responsible Design, Implementation and Use of Information and Communication Technology, 12067, 223-234. https://doi.org/10.1007/978-3-030-45002-1_19.

Cornwall, W. (2020). Officials gird for a war on vaccine misinformation. Fears of a rushed COVID-19 vaccine and rise of social media demand new messaging strategy. Science, 369(6499), 14-15.

Dawson, R., Lemmon, K., Trivedi, N. J., \& Hansen, S. (2018). Improving human papilloma virus vaccination rates throughout military treatment facilities. Vaccine, 36(11), 1361-1367.

Dempsey, A., Pyrznawoski, J., Lockhart, S., Barnard, J., Campagna, E. J., Garrett, K., Fisher, A., Dickinson, L. M., \& O'Leary, S. T. (2018). Effect of a health care professional communication training intervention on adolescent human papillomavirus vaccination. A cluster randomized clinical trial. JAMA Pediatrics, 172(5), e180016. https:// doi.org/10.1001/jamapediatrics.2018.0016.

Dubé, E., Gagnon, D., \& Vivion, M. (2020). Optimizing communication material to address vaccine hesitancy. Canada Communicable Disease Report, 46(2/3), 48-52.

Gagneur, A., Gosselin, V., \& Dubé, E. (2018). Motivational interviewing: a promising tool to address vaccine. Vaccine, 36(44), 6553-6555.

Gold, M. S., MacDonald, N. E., McMurtry, M. C., Balakrishnan, M. R., Heininger, U., Menning, L., Benes, O., Pless, R., \& Zuber, P. L. F. (2020). Immunization stress-related response - redefining immunization anxiety-related reaction as an adverse event following immunization. Vaccine, 38(14), 3015-3020.

Graven, M., Allen, P., Smith, I., \& MacDonald, N. E. (2013). Decline in mortality with the Belize integrated patient-centred country wide health information system (BHIS) with embedded program management. International Journal of Medical Informatics, 82(10), 954-963.

Hampton, L. M. (2020). Vaccine handling and administration errors should be addressed to improve vaccine program safety. Vaccine, 38(32), 4933-4934.

Hibbs, B. F., Moro, P. L., Miller, E. R., \& Shimabukuro, T. T. (2015). Vaccination errors reported to vaccine adverse event reporting system (VAERS United States, 2000-2013). Vaccine, 33(28), 31713178 .
Hosangadi, D., Warmbrod, K. L., Martin, E. K., Adalja, A., Cicero, A., Inglesby, T., Watson, C., Watson, M., \& Connell, N. (2020). Enabling emergency mass vaccination: innovations in manufacturing and administration during a pandemic. Vaccine, 38(26), 41674169 .

Lane, S., MacDonald, N. E., Marti, M., \& Dumolard, L. (2018). Vaccine hesitancy around the globe: a three year assessment of data from the annual WHO/UNICEF Joint Reporting Form. Vaccine, 36(26), 3861-3867.

Lurie, N., Saville, M., Hatchette, R., \& Halton, J. (2020). Developing Covid-19 vaccines at pandemic speed. The New England Journal of Medicine, 382(21), 1969-1973.

MacDonald, N. E., \& Dubé, E. (2020). Promoting immunization resiliency in the digital information age. Canada Communicable Disease Report, 46(1), 20-24.

MacDonald, N. E., \& Law, B. J. (2017). Canada's eight-component vaccine safety system: a primer for health care workers. Paediatrics \& Child Health, 22(4), e13-e16.

MacDonald, N. E., Comeau, J. L., Dubé, E., \& Bucci, L. (2020a). COVID-19 and missed routine immunizations: designing for effective catch-up in Canada. Canadian Journal of Public Health, 111, 469-472.

MacDonald, N. E., Guichard, S., Arora, N., Menning, L., Wilhelm, E., \& Participants, 2019 Inter-country SEAR Workshop. (2020b). Lessons on causality assessment and communications from the 2019 South-East Asia Regional (SEAR) workshop on intercountry expert review of selected adverse events following immunization (AEFI) cases. Vaccine, 38(32), 4924-4932.

Mostafapour, M., Meyer, S. B., \& Scholer, A. (2019). Exploring the effect of risk and benefit information provision on vaccination decision-making. Vaccine, 37(44), 6750-6759.

Nichols, M. K., Andrew, M. K., Hatchette, T. F., Ambrose, A., Boivin, G., Bowie, W., et al. (2018). Influenza vaccine effectiveness to prevent influenza-related hospitalizations and serious outcomes in Canadian adults over the 2011/12 through 2013/14 influenza seasons: a pooled analysis from the (CIRN) Serious Outcomes Surveillance Network. Vaccine, 36(16), 2166-2175.

Pltokin, S. A., Mahmoud, A. A. F., \& Farrar, J. (2015). Establishing a global vaccine-development fund. The New England Journal of Medicine, 373, 297-300.

Randolph, H. E., \& Barreiro, L. B. (2020). Herd immunity: understanding COVID-19. Immunity, 52(5), 737-741.

Ryan, M. J., Giles-Vernick, T., \& Graham, J. E. (2019). Technologies of trust in epidemic response: openness, reflexivity and accountability during the 2014-2016 Ebola outbreak in West Africa. BMJ Global Health, 4, e001272. https://doi.org/10.1136/bmjgh-2018-001272.

Sadique, M. Z., Devlin, N., Edmunds, W. J., \& Parkin, D. (2013). The effect of perceived risks on the demand for vaccination: results from a discrete choice experiment. PLoS One, 8(2), e5149. https://doi.org/ 10.1371/journal.pone.0054149.

Schmid, P., \& Betsch, C. (2019). Effective strategies for rebutting science denialism in public discussions. Nature Human Behaviour, 3, 931939. Retrieved from: https://doi.org/10.1038/s41562-019-0632-4.

Shelby, A., \& Ernst, K. (2013). Story and science. How providers and parents can utilize storytelling to combat anti-vaccine movement. Human Vaccines \& Immunotherapeutics, 9, 1795-1801.

Steenbeek, A., MacDonald, N. E., Downie, J., Appleton, M., \& Baylis, F. (2012). Ill informed consent: a content analysis of the physical risk disclosure in school-based HPV vaccine programs: the Canadian experience. Public Health Nursing, 29, 71-79.

Top, K. A., Billard, M. N., Gariepy, M. C., Rouleau, I., Pernica, J. M., Pham-Huy, A., Quach, C., Tran, D., Vaudry, W., Dobson, S., Boucher, F. D., Carignan, A., Jadavji, T., McConnell, A., McNeil, S. A., Halperin, S. A., De Serres, G., \& Investigators, CIRN Special Immunization Clinic Network. (2016). Immunizing patients with adverse events after immunization and potential contraindications 
to immunization: a report from the Special Immunization Clinics Network. The Pediatric Infectious Disease Journal, 35(12), e384 e391.

Usher, A. D. (2020). COVID-19 vaccines for all? Lancet, 395(10240), 1822-1823.

van der Linden, S., Maibach, E., Cook, J., Leiserowitz, A., \& Lewandowsky, S. (2017, Dec 1). Inoculating against misinformation. Science, 358(6367), 1141-1142.
World Health Organization, Strategic Advisory Group of Experts on Immunization. (2020). WHO SAGE values framework for the allocation and prioritization of COVID-19 vaccination, 14 September 2020. Retrieved September 15, 2020, from World Health Organization: https://apps.who.int/iris/handle/10665/334299.

Publisher's note Springer Nature remains neutral with regard to jurisdictional claims in published maps and institutional affiliations. 